\title{
Thermal conductivity of a granular metal
}

\author{
V. Tripathi and Y. L. Loh \\ Theory of Condensed Matter Group, Cavendish Laboratory, \\ Department of Physics, University of Cambridge, \\ Madingley Road, Cambridge CB3 OHE, United Kingdom
}

(Dated: 10th November 2018)

\begin{abstract}
Using the Kubo formula approach, we study the effect of electron interaction on thermal transport in the vicinity of a metal-insulator transition, with a granular metal as our model. For small values of dimensionless intergrain tunneling conductance, $g \ll 1$, we find that the thermal conductivity surprisingly shows a phonon-like algebraic decrease, $\kappa(T) \sim g^{2} T^{3} / E_{c}^{2}$ even though the electrical conductivity obeys an Arrhenius law, $\sigma(T) \sim g e^{-E_{c} / T}$; therefore the Wiedemann-Franz (WF) law is seriously violated. We explicitly show that this violation arises from non-magnetic bosonic excitations of low energy that transport heat but not charge. At large values of intergrain tunneling, we find it plausible that the WF law weakly deviates from the free-electron theory due to potential fluctuations. Implications for experiment are discussed.
\end{abstract}

At low temperatures, transport in many metals is dominated by elastic scattering of electrons. In the absence of phase transitions (such as superconductivity) and collective modes, the Wiedemann-Franz (WF) law 1] relates the electrical $(\sigma)$ and thermal $(\kappa)$ conductivities through a universal Lorenz number, $L_{0}=\frac{\kappa(T)}{T \sigma(T)}$ where $L_{0} \equiv \frac{\pi^{2} k_{B}^{2}}{3 e^{2}}$ is Sommerfeld's value for the Lorenz number. Deviations from the Wiedemann-Franz (WF) law beyond those due to phonons and inelastic processes such as electronphonon scattering indicate a presence of collective modes that discriminate between charge and energy flow. Such deviations are expected to get more pronounced as one approaches a metal-insulator transition.

The issue of excess electronic thermal conductivity in disordered metals with interacting electrons has been a topic of much theoretical debate in recent years. The WF law has been affirmed for any strength of scattering [2], disorder [3], or magnetic field [4]. In Refs. 5, 6], the WF law was shown to hold on account of a Ward identity. Subsequently there have been many studies including the effect of electron interaction, some of which have suggested deviations from the WF law [7, 8, 9, 10, 11, 12].

In this paper we study the effect of Coulomb blockade on the thermal conductivity of a nonmagnetic granular metal. Granular metals are a novel playground, experimentally and theoretically, for studying electron interaction effects in the vicinity of a metal-insulator transition. At not too low temperatures [13] transport in a granular metal is due to a competition of incoherent intergrain tunneling and Coulomb blockade [13, 14]. The resulting model captures the physics of coupling of the continuum of electron states in the metallic grains with electromagnetic fluctuations. Granular metals differ from Hubbard models in having a near-continuum of excitations in each metallic grain. Their study is relevant beyond artificially-prepared granular metals [15, 16]. For example, in more insulating regimes, these materials show a similar electrical conductivity to disordered semiconductors in the VRH regime. In more conducting regimes, granular metal physics appears to describe transport in underdoped cuprate superconductors [17, 18] where there is electronic phase separation into metallic and insulating regions [19].

We summarize our main results. We obtain for the first time an explicit Kubo formula for the thermal conductivity of the AES model through a double functional differentiation of the partition function with respect to an appropriate source field 20]. For small values of the dimensionless intergrain conductance, $g \ll 1$, we identify lowenergy non-magnetic bosonic excitations that contribute to heat transport but not charge transport. Consequently, as our calculations show, the thermal conductivity decreases only algebraically, $\kappa(T) \sim g^{2} T^{3} / E_{c}^{2}$, quite unlike the electrical conductivity of a regular granular array, which obeys an Arrhenius law, $\sigma(T) \sim g e^{-E_{c} / T}$. These bosonic modes are the nearly-continuous particlehole excitations in the metallic grains and have no counterpart in a half-filled Hubbard model with $U \sim E_{c}$ where particle-hole excitations are gapped. Since the electrical conductivity is insensitive to these neutral excitations, the algebraic thermal conductivity we calculate distinguishes between a granular metal and a Hubbard model. This finding also reminds us of the excess heat transport through excitons proposed recently [21, 22] in the context of disordered semiconductors. For strong tunneling, $g \gg 1$, we find it plausible that the Lorenz number weakly deviates from the Sommerfeld value $L_{0}$ due to Coulomb interaction.

Consider the following microscopic Hamiltonian for a granular metal array ignoring spin interactions,

$$
\begin{aligned}
H= & \sum_{\mathbf{x} \lambda}\left[\psi_{\mathbf{x} \lambda}^{\dagger} \xi_{\mathbf{x} \lambda} \psi_{\mathbf{x} \lambda}+E_{c} \sum_{\mathbf{x}}\left(\hat{Q}_{\mathbf{x}}-Q_{0}\right)^{2}\right]\left(1+h_{\mathbf{x}}\right)+ \\
+ & \frac{1}{2} \sum_{\left\langle\mathbf{x} \mathbf{x}^{\prime}\right\rangle, \lambda \lambda^{\prime}}\left(t_{\mathbf{x} \mathbf{x}^{\prime}}^{\lambda \lambda^{\prime}} \psi_{\mathbf{x} \lambda}^{\dagger} \psi_{\mathbf{x}^{\prime} \lambda^{\prime}}+\text { h.c. }\right)\left(1+h_{\mathbf{x}}\right)
\end{aligned}
$$

where $\mathbf{x}$ labels the grains and $\lambda$ labels the states within each grain, $\xi_{\mathbf{x} \lambda}=\epsilon_{\mathbf{x} \lambda}-\mu . \hat{Q}_{\mathbf{x}}=\sum_{\lambda} \psi_{\mathbf{x} \lambda}^{\dagger} \psi_{\mathbf{x} \lambda}$ is the charge 
on grain $\mathbf{x}, E_{c}$ is the grain charging energy, and $t_{\mathbf{x} \mathbf{x}^{\prime}}^{\lambda \lambda^{\prime}}$ is the matrix element for nearest-neighbor hopping. Small differences in temperature of different grains are modeled through the introduction of "gravitational" potentials $h_{\mathbf{x}}$ 20] that couple to the grain electron energy. If $\beta=1 / T$ is the average inverse temperature, then $h_{\mathbf{x}}=\left(\beta_{\mathbf{x}}-\beta\right)$ is the small excess of $\beta$ on the $\mathbf{x}^{\text {th }}$ grain.

The strong Coulomb interaction in Eq.(1) makes it difficult to calculate correlation functions directly using the electron operators. We therefore decouple the interaction to obtain an action for a Hubbard-Stratonovich field, $V$, representing the electrostatic potential on each grain,

$$
\begin{aligned}
& S_{0}[V]=-\frac{1}{4 E_{c}} \sum_{\mathbf{x}} \int_{0}^{\beta} d \tau \frac{V_{\tau \mathbf{x}}^{2}}{1+h_{\tau \mathbf{x}}}+ \\
& +\sum_{\mathbf{x} \mathbf{x}^{\prime} ; \lambda \lambda^{\prime}} \int_{0}^{\beta} d \tau t_{\mathbf{x} \mathbf{x}^{\prime}}^{\lambda \lambda^{\prime}} \psi_{\mathbf{x} \lambda}^{\dagger} \psi_{\mathbf{x}^{\prime} \lambda^{\prime}}\left(1+\frac{h_{\tau \mathbf{x}}+h_{\tau \mathbf{x}^{\prime}}}{2}\right)+ \\
& +\sum_{\mathbf{x} \lambda} \int_{0}^{\beta} d \tau \psi_{\mathbf{x} \lambda}^{\dagger}\left(\partial_{\tau}+V_{\tau \mathbf{x}}+\xi_{\mathbf{x} \lambda}\left(1+h_{\tau \mathbf{x}}\right)\right) \psi_{\mathbf{x} \lambda}
\end{aligned}
$$

where $\tau$ is imaginary time, and the $h_{\mathbf{x}}$ sources are now $\tau$-dependent. We then make the gauge transformation $\psi_{\mathbf{x} \lambda} \rightarrow e^{-i \varphi_{\tau \mathbf{x}}} \psi_{\mathbf{x} \lambda}$ and $V_{\mathbf{x}}=i \partial_{\tau} \varphi_{\tau \mathbf{x}}$. This eliminates the $\psi^{\dagger} V \psi$ term, at the cost of replacing $t$ by a gaugedependent hopping amplitude, $\tilde{t}_{\tau \mathbf{x x}^{\prime}}^{\lambda \lambda^{\prime}}=t_{\mathbf{x} \mathbf{x}^{\prime}}^{\lambda \lambda^{\prime}} e^{i \varphi_{\tau \mathbf{x} \mathbf{x}^{\prime}}}$, where $\varphi_{\tau \mathbf{x x}^{\prime}}=\varphi_{\tau \mathbf{x}}-\varphi_{\tau \mathbf{x}^{\prime}}$. The Matsubara fields $\varphi_{\tau \mathbf{x}}$ satisfy bosonic boundary conditions, $\varphi_{\beta \mathbf{x}}=2 \pi k_{\mathbf{x}}+\varphi_{0 \mathbf{x}}$, where $k_{\mathbf{x}} \in \mathbb{Z}$ is the winding number at site $\mathbf{x}$.

Next we integrate out the fermions and expand the electron determinant to $O\left(h \tilde{t}^{2}\right)$,

$$
\begin{array}{r}
S[\varphi, h] \approx \frac{1}{4 E_{c}} \sum_{\mathbf{x}} \int_{\tau} \frac{\left(\partial_{\tau} \varphi_{\tau \mathbf{x}}\right)^{2}}{1+h_{\tau \mathbf{x}}}+\operatorname{tr}\left[G_{\mathbf{x} \lambda}(\tau, \tau) h_{\tau \mathbf{x}} \xi_{\mathbf{x} \lambda}\right]+ \\
+\frac{1}{2} \operatorname{tr}\left[G_{\mathbf{x} \lambda}\left(\tau_{1}, \tau_{2}\right) \tilde{t}_{\tau_{2} \mathbf{x} \mathbf{x}^{\prime}}^{\lambda \lambda^{\prime}} G_{\mathbf{x}^{\prime} \lambda^{\prime}}\left(\tau_{2}, \tau_{1}\right) \tilde{t}_{\tau_{1} \mathbf{x}^{\prime} \mathbf{x}}^{\lambda^{\prime} \lambda}\right]+ \\
+\frac{1}{2} \operatorname{tr}\left[G_{\mathbf{x} \lambda}\left(\tau_{1}, \tau_{2}\right) \tilde{t}_{\tau_{2} \mathbf{x} \mathbf{x}^{\prime}}^{\lambda \lambda^{\prime}} G_{\mathbf{x}^{\prime} \lambda^{\prime}}\left(\tau_{2}, \tau_{1}\right) \tilde{t}_{\tau_{1} \mathbf{x}^{\prime} \mathbf{x}}^{\lambda^{\prime} \lambda}\left(h_{\tau_{2} \mathbf{x}}+h_{\tau_{2} \mathbf{x}^{\prime}}\right)\right]+ \\
+\operatorname{tr}\left[G_{\mathbf{x} \lambda}\left(\tau_{1}, \tau_{2}\right) h_{\tau_{2} \mathbf{x}} \xi_{\mathbf{x} \lambda} G_{\mathbf{x} \lambda}\left(\tau_{2}, \tau_{3}\right) \tilde{t}_{\tau_{3} \mathbf{x} \mathbf{x}^{\prime}}^{\lambda \lambda^{\prime}} G_{\mathbf{x}^{\prime} \lambda^{\prime}}\left(\tau_{3}, \tau_{1}\right) \tilde{t}_{\tau_{1} \mathbf{x}^{\prime} \mathbf{x}}^{\lambda^{\prime} \lambda}\right.
\end{array}
$$

where "tr" means a sum over all indices, and $G_{\mathbf{x} \lambda}\left(\tau, \tau^{\prime}\right)$ is the electron Green function

$G_{\mathbf{x} \lambda}\left(\tau, \tau^{\prime}\right)=T \sum_{n} \frac{e^{-i \nu_{n}\left(\tau-\tau^{\prime}\right)}}{i \nu_{n}-\xi_{\mathbf{x} \lambda}}=\left[n\left(\xi_{\mathbf{x} \lambda}\right)-\Theta_{\tau-\tau^{\prime}}\right] e^{-\xi_{\mathbf{x} \lambda}\left(\tau-\tau^{\prime}\right)}$.

Here $\nu_{n}=2 \pi T(n+1 / 2)$ and $\Theta_{\tau}$ is the unit step function. We will also need the momentum-summed Green function, $G\left(\tau, \tau^{\prime}\right)=\sum_{\lambda} G_{\mathbf{x} \lambda}\left(\tau, \tau^{\prime}\right)$, which is independent of the grain label. If the temperature is much larger than the Thouless energy for intergrain diffusion, $T \gg|t|^{2} \nu\left(\epsilon_{F}\right)$, and if the grains are much larger than the Fermi wavelength of the metal, it suffices to expand the electron determinant to second order in tunneling [23].

The (time-dependent) energy of the $\mathbf{x}^{t h}$ grain, $E_{\tau \mathbf{x}}$, is obtained by differentiating the action in Eq. (3) with respect to $h_{\tau \mathbf{x}}$. Dropping any constant terms, and using the above Green functions, we have,

$$
\begin{aligned}
& E_{\tau \mathbf{x}}=-\frac{1}{4 E_{c}}\left(\partial_{\tau} \varphi_{\tau \mathbf{x}}\right)^{2}+\sum_{\mathbf{x}^{\prime}=\mathbf{x} \pm \mathbf{a}}|t|^{2}\left[\int_{\tau_{1}} G\left(\tau_{1}, \tau\right) \times\right. \\
& \times G\left(\tau, \tau_{1}\right) \cos \left(\varphi_{\tau \mathbf{x} \mathbf{x}^{\prime}}-\varphi_{\tau_{1} \mathbf{x} \mathbf{x}^{\prime}}\right)+\sum_{\lambda \lambda^{\prime}} \int_{\tau_{1} \tau_{2}} \xi_{\mathbf{x} \lambda} G_{\mathbf{x} \lambda}\left(\tau_{1}, \tau\right) \times \\
& \left.\times \quad G_{\mathbf{x} \lambda}\left(\tau, \tau_{2}\right) G_{\mathbf{x}^{\prime} \lambda^{\prime}}\left(\tau_{2}, \tau_{1}\right) \exp \left[i\left(\varphi_{\tau_{2} \mathbf{x x}^{\prime}}-\varphi_{\tau_{1} \mathbf{x} \mathbf{x}^{\prime}}\right)\right]\right](5)
\end{aligned}
$$

where we assumed that the bare tunneling element is a constant. To obtain an expression for the energy current $J_{\tau \mathbf{x x}^{\prime}}$ from grain $\mathbf{x}$ to a neighboring grain $\mathbf{x}^{\prime}$, we employ the continuity equation, $\partial_{\tau} E_{\tau \mathbf{x}}=i \sum_{\mathbf{x}^{\prime}} J_{\tau \mathbf{x x}^{\prime}}$. We will need the equation of motion for $\varphi_{\tau \mathbf{x}}$ from Eq. (3) (with sources set to zero) and the Green functions defined in Eq. (4). The symmetrized energy current, $j_{\tau \mathbf{x x}^{\prime}}^{(E)}=$ $\frac{1}{2}\left(J_{\tau \mathbf{x} \mathbf{x}^{\prime}}-J_{\tau \mathbf{x}^{\prime} \mathbf{x}}\right)$, is

$$
\begin{aligned}
& j_{\tau \mathbf{x} \mathbf{x}^{\prime}}^{(E)}=i \pi g T^{2} \int_{\tau^{\prime}} \frac{1}{2}\left(\partial_{\tau} \varphi_{\tau \mathbf{x}}+\partial_{\tau} \varphi_{\tau \mathbf{x}^{\prime}}\right) \frac{1}{\sin ^{2}\left[\pi T\left(\tau-\tau^{\prime}\right)\right]} \times \\
& \times \sin \left(\varphi_{\tau \mathbf{x} \mathbf{x}^{\prime}}-\varphi_{\tau^{\prime} \mathbf{x} \mathbf{x}^{\prime}}\right)-\pi g T^{2} \int_{\tau^{\prime}}\left[\partial_{\tau} \frac{1}{\sin \left[\pi T\left(\tau-\tau^{\prime}\right)\right]}\right] \times \\
& \times \frac{1}{\sin \left[\pi T\left(\tau-\tau^{\prime}\right)\right]} \sin \left(\varphi_{\tau \mathbf{x} \mathbf{x}^{\prime}}-\varphi_{\tau^{\prime} \mathbf{x} \mathbf{x}^{\prime}}\right)
\end{aligned}
$$

where we have introduced the dimensionless intergrain tunneling conductance $g=2 \pi \nu^{2}\left(\epsilon_{F}\right)|t|^{2}$ and used $G\left(\tau, \tau^{\prime}\right)=\frac{\pi \nu\left(\epsilon_{F}\right) T}{\sin \pi T\left(\tau-\tau^{\prime}\right)}$. To obtain the Kubo formula for thermal conductivity, we need to calculate the non-local specific heat through a double differentiation of the partition function with respect to $h_{\tau \mathbf{x}}$, and then extract the thermal conductivity using the continuity equation.

The same result may be obtained more easily by introducing a source field $f_{\tau \mathbf{x}}$ that can generate the energy (3) current, $h_{\tau \mathbf{x}}=i \partial_{\tau} f_{\tau \mathbf{x}}$. In Eq.(2) we expand to linear order in $h_{\tau \mathbf{x}}$ and use the field $f_{\tau \mathbf{x}}$ instead of $h_{\tau \mathbf{x}}$. Next we integrate out the conduction electrons and expand to second order in tunneling. The resulting action is

$$
\begin{aligned}
S[\varphi, f] \approx & \frac{1}{4 E_{c}} \sum_{\mathbf{x}} \int_{\tau}\left(\partial_{\tau} \varphi_{\tau \mathbf{x}}\right)^{2}+ \\
& +\frac{1}{2} \operatorname{tr}\left[G_{\mathbf{x} \lambda}\left(\tau_{1}, \tau_{2}\right) \hat{t}_{\tau_{2} \mathbf{x} \mathbf{x}^{\prime}}^{\lambda \lambda^{\prime}} G_{\mathbf{x}^{\prime} \lambda^{\prime}}\left(\tau_{2}, \tau_{1}\right) \hat{t}_{\tau_{1} \mathbf{x}^{\prime} \mathbf{x}}^{\lambda^{\prime} \lambda}\right. \\
\hat{t}_{\tau \mathbf{x} \mathbf{x}^{\prime}}^{\lambda \lambda^{\prime}}= & \tilde{t}_{\tau \mathbf{x} \mathbf{x}^{\prime}}^{\lambda \lambda \lambda^{\prime}}\left[1+i f_{\tau \mathbf{x} \mathbf{x}^{\prime}}\left(\left(\xi_{\mathbf{x} \lambda}+\xi_{\mathbf{x}^{\prime} \lambda^{\prime}}\right) / 2+V_{\tau \mathbf{x} \mathbf{x}^{\prime}}^{(a v)}\right)\right]
\end{aligned}
$$

where $f_{\tau \mathbf{x} \mathbf{x}^{\prime}}=f_{\tau \mathbf{x}}-f_{\tau \mathbf{x}^{\prime}}$ and $V_{\tau \mathbf{x} \mathbf{x}^{\prime}}^{(a v)}=\left(V_{\tau \mathbf{x}}+V_{\tau \mathbf{x}^{\prime}}\right) / 2$. The energy current in Eq.(6) can be obtained from Eq.(7) using $j_{\tau \mathbf{x} \mathbf{x}^{\prime}}^{(E)}[\varphi, f]=\delta S[\varphi, f] / \delta f_{\tau \mathbf{x x}^{\prime}}$. This is similar to the derivation of the electric current, with electric charge replaced by electronic energy. The thermal conductivity $\kappa$ 
may be obtained by further differentiation,

$$
\begin{aligned}
\kappa(\omega, T) & =\left.i a^{2-d} \frac{1}{\omega} \int_{0}^{\beta} d \tau e^{i \Omega_{n} \tau} K^{(E)}(\tau)\right|_{\Omega_{n} \rightarrow-i \omega},(9) \\
K^{(E)}\left(\tau_{1}-\tau_{2}\right) & =\frac{\beta}{Z} \int D \varphi e^{-S}\left[\frac{\delta j_{\tau_{2} \mathbf{x}, \mathbf{x}+\mathbf{a}}^{(E)}[\varphi, f]}{\delta f_{\tau_{1} \mathbf{x}, \mathbf{x}+\mathbf{a}}}-\right. \\
& \left.-\sum_{\mathbf{x}^{\prime}} \int j_{\tau_{2} \mathbf{x}^{\prime}, \mathbf{x}^{\prime}+\mathbf{a}}^{(E)} \frac{\delta S[\varphi, f]}{\delta f_{\tau_{1} \mathbf{x}^{\prime}, \mathbf{x}^{\prime}+\mathbf{a}}}\right]\left.\right|_{f=0},(10)
\end{aligned}
$$

where $\omega^{+}=\omega+i \delta$. The first term, which has come to be known as the "diamagnetic" contribution $\kappa^{(d)}$, is a local term in the sense that it is an average on a single bond. The second term in Eq.(10) is a product of two energy currents, and is also known as the "paramagnetic" contribution $\kappa^{(p)}$. The diamagnetic contribution may be obtained by differentiating Eq. (17) twice with respect to the source,

$$
\begin{aligned}
& \kappa^{(d)}(\omega, T)=-i a^{2-d} \frac{\pi^{2} g T^{3}}{\omega} \int_{0}^{\beta} d \tau\left(e^{i \Omega_{n} \tau}-1\right) \times \\
& {\left[\frac{1}{\sin \pi T \tau} \frac{\partial^{2}}{\partial_{\tau_{c}}^{2}} \frac{1}{\sin \pi T\left(\tau+i \tau_{c}\right)}-V_{\tau \mathbf{x}, \mathbf{x}+\mathbf{a}}^{(a v)} V_{0 \mathbf{x}, \mathbf{x}+\mathbf{a}}^{(a v)} \frac{1}{\sin ^{2} \pi T \tau}\right]} \\
& \times\left.\left\langle\cos \left(\varphi_{\tau \mathbf{x}, \mathbf{x}+\mathbf{a}}-\varphi_{0 \mathbf{x}, \mathbf{x}+\mathbf{a}}\right)\right\rangle\right|_{\Omega_{n} \rightarrow-i \omega^{+}, \tau_{c} \rightarrow 0} \cdot
\end{aligned}
$$

Let us call the $V$-independent part of Eq. (111) the kinetic contribution, $\kappa^{(d, K)}$, and the $V$-dependent part the potential contribution $\kappa^{(d, V)}$. Eq. (11) is similar to the known expression for the diamagnetic contribution to the electrical conductivity obtained in Ref. 24].

A. Consider first weakly coupled grains, $g \ll 1$. Do a perturbation expansion in $g, \kappa=\kappa^{(1)}+\kappa^{(2)}+\cdots$, where $\kappa^{(n)} \sim g^{n}$. The diamagnetic parts of the electrical 24] and thermal conductivities [Eq. 11] involve the bond correlator, $\Pi_{\tau}=\left\langle\exp i\left(\varphi_{\tau \mathbf{x}, \mathbf{x}+\mathbf{a}}-\varphi_{0 \mathbf{x}, \mathbf{x}+\mathbf{a}}\right)\right\rangle=\Pi^{(0)}+$ $\Pi^{(1)}+\cdots$. Because of the Coulomb blockade, $\Pi_{\tau}^{(0)}=$ $\frac{1}{Z} \sum_{q_{1}, q_{2}} e^{-\left(q_{1}^{2}+q_{2}^{2}\right) \beta E_{c}-2 E_{c} \tau\left(1-q_{1}-q_{2}\right)}$ is exponential in $\tau$.
So

$$
\begin{aligned}
\sigma^{(d, 1)} & =g e^{2} a^{2-d}\left(2 e^{-\beta E_{c}}+2 \beta E_{c} e^{-2 \beta E_{c}}\right), \\
\kappa^{(d, K, 1)} & =g T a^{2-d}\left(\frac{2 \pi^{2}}{3} e^{-\beta E_{c}}+\frac{8}{3}\left(\beta E_{c}\right)^{3} e^{-2 \beta E_{c}}\right) .
\end{aligned}
$$

In contrast, $\Pi_{\tau}^{(1)}$ has a power-law $\tau$-dependence 25], which is a result of the $1 / \tau^{2}$ interaction in the AES action, Eq.(7), arising from particle-hole inelastic cotunneling:

$$
\Pi_{\tau}^{(1)}=\frac{2 \pi g T^{2}}{E_{c}^{2} \sin ^{2}(\pi T \tau)}=\frac{2 \pi g}{E_{c}^{2}} \alpha_{\tau}, \quad \alpha_{\tau}=\frac{T^{2}}{\sin ^{2}(\pi T \tau)} .
$$

It gives rise to $O\left(g^{2}\right)$ terms in both the diamagnetic and paramagnetic contributions to $\sigma$ and $\kappa$. We have shown
[25] in a previous work that

$$
\begin{aligned}
\sigma^{(d, 2)} & =-\sigma^{(p, 2)}=\left.\frac{i \pi g a^{2-d} e^{2}}{\omega}\left(\frac{2 \pi g}{E_{c}^{2}}\right) \int_{\tau}\left(e^{i \Omega_{n} \tau}-1\right) \alpha_{\tau}^{2}\right|_{\Omega_{n} \rightarrow-i \omega^{+}} \\
& \approx \frac{4 \pi}{3} e^{2} a^{2-d}\left(\frac{g T}{E_{c}}\right)^{2}
\end{aligned}
$$

the power-law terms in the electrical conductivity cancel 25], leaving an Arrhenius law $\sigma=\sigma^{(d, 1)} \sim e^{-E_{c} / T}$. Now consider the $O\left(g^{2}\right)$ terms in the thermal conductivity. The dominant contributions to both the diamagnetic and paramagnetic terms arise from charge-neutral configurations $\left(q_{1}=q_{2}=0\right)$, and, unlike the $O(g)$ terms, are not Arrhenius-suppressed:

$$
\begin{aligned}
& \kappa^{(d, K, 2)}=\left.\frac{i \pi g a^{2-d}}{\omega}\left(\frac{2 \pi g}{T E_{c}^{2}}\right) \int_{\tau}\left(e^{i \Omega_{n} \tau}-1\right) \gamma_{\tau} \alpha_{\tau}\right|_{\Omega_{n} \rightarrow-i \omega^{+}}, \\
& \kappa^{(p, K, 2)}=\left.\frac{i \pi g a^{2-d}}{4 \omega}\left(\frac{2 \pi g}{T E_{c}^{2}}\right) \int_{\tau}\left(e^{i \Omega_{n} \tau}-1\right) \alpha_{\tau}^{\prime} \alpha_{\tau}^{\prime}\right|_{\Omega_{n} \rightarrow-i \omega^{+}},
\end{aligned}
$$

where $\gamma_{\tau}=T^{2}(\sin (\pi T \tau))^{-1} \partial_{\tau}^{2}\left(\sin (\pi T \tau)^{-1}\right.$. These two terms have the same sign and do not cancel,

$$
\kappa^{(d, K, 2)}=3 \kappa^{(p, K, 2)}=\frac{12 \pi^{3}}{15} a^{2-d}\left(\frac{g^{2} T^{3}}{E_{c}^{2}}\right),
$$

and so the thermal conductivity is only algebraically small,

$$
\kappa^{(K, 2)}=\left(16 \pi^{3} / 15\right)\left(g^{2} T^{3} / E_{c}^{2}\right) .
$$

This is the central result of our paper. In the argument above, the algebraic temperature dependence is an eventual result of the particle-hole inelastic cotunneling processes described by the AES action.

The potential energy contributions $\kappa^{(V, 2)}$ and $\kappa^{(V, 2)}$, which involve averages such as $\left\langle V_{\tau \mathbf{x}, \mathbf{x}+\mathbf{a}}^{(a v)} V_{0 \mathbf{x}, \mathbf{x}+\mathbf{a}}^{(a v)} \cos \left(\varphi_{\tau \mathbf{x}, \mathbf{x}+\mathbf{a}}-\varphi_{0 \mathbf{x}, \mathbf{x}+\mathbf{a}}\right)\right\rangle$, turn out to be proportional to $\left(q_{1}-q_{2}\right)^{2}$. This immediately rules out neutral processes, such as inelastic cotunneling, for which $q_{1}=q_{2}=0$. The $O\left(g^{0}\right)$ contribution to the above average can be shown to be $E_{c}^{2} \sum_{q_{1}, q_{2}}\left(q_{1}-q_{2}\right)^{2} e^{-\left(q_{1}^{2}+q_{2}^{2}\right) \beta E_{c}-2 E_{c} \tau\left(1-q_{1}-q_{2}\right)}$, so that $\kappa^{(V, 2)} \sim O\left(e^{-\beta E_{c}}\right)$, which can be neglected in comparison with $\kappa^{(K, 2)}$.

B. Consider now the case where the dimensionless intergrain tunneling conductance $g$ is large, $g=$ $\pi|t|^{2} \nu\left(\epsilon_{F}\right)^{2}$. One can show 24 that the diamagnetic contribution to the electrical conductivity is $\sigma^{(d)}=$ $e^{2} a^{2-d} g\left(1-\frac{1}{\pi g z} \ln \frac{g E_{c}}{T}\right)$. Comparing with Eq. (111), it is evident that for the diamagnetic components, the kinetic part of the thermal conductivity is simply related to the electrical conductivity by $\kappa^{(d, K)} /\left(T \sigma^{(d)}\right)=\frac{\pi^{2} k_{B}^{2}}{3 e^{2}}$, where we have restored the Boltzmann constant $k_{B}$. So at this 
level the WF law is obeyed. Next consider the kinetic part of the paramagnetic contribution to the thermal conductivity, $\kappa^{(p, K)}(T)$. Ref. 24 shows that $\sigma^{(p)} \ll \sigma^{(d)}$; adopting a similar argument, one can show that $\kappa^{(p, K)}$ is smaller than $\kappa^{(d, K)}$ by a factor $g^{-2}$, and furthermore it does not have a logarithmic singularity. We have not yet considered the extra contributions from the potential part, $\kappa^{(d, V)}$ and $\kappa^{(p, V)}$. Evaluating $\kappa^{(d, V)}$ for example leads to corrections similar to those obtained in Ref. 12]. However since the potential part $\kappa^{(p, V)}$ of the paramagnetic term is of the order of $\kappa^{(d, V)}$, a proper treatment should consider both contributions. This could, in principle, lead to a different conclusion from Ref. [12]. So at this stage we are only able to make a weaker statement that for the strong tunneling case at not too low temperatures $\left(T \gg g E_{c} e^{-\pi g z}\right), \frac{\kappa}{T \sigma}=\frac{\pi^{2} k_{B}^{2}}{3 e^{2}}+O\left(g^{-1}\right)$. Thus the Lorenz number $L$ is larger than Sommerfeld's free electron value by $O\left(g^{-1}\right)$ corrections.

We conclude with a discussion of our results and comments on existing and future experiment. We have shown that thermal transport in granular metals at low temperatures is dominated by cotunneling of lowenergy electron-hole pairs. These neutral excitations do not transport charge. As a result, while the electrical conductivity for weakly coupled grains is exponentially small in temperature, $\sigma(T) \sim e^{2} g a^{2-d} e^{-E_{c} / T}$, the thermal conductivity is only algebraically small, $\kappa(T) \sim$ $a^{2-d} k_{B}^{2} g^{2} T^{3} / E_{c}^{2}$. The particle-hole cotunneling process is physically equivalent to a particle cotunneling loop. An electron executing a cotunneling loop brings back its charge to the starting grain and hence there is little change in the electrical conductivity. There is no requirement, however, that the returning electron has exactly the same energy. This conservation of grain charge but not grain energy in a cotunneling loop is at the heart of the difference between heat and charge transport. The energy gained by an electron due to nearest neighbor tunneling is of the order of $\left(t^{2} / E_{c}\right)$, and the tunneling probability is, roughly, $\left(t / E_{c}\right)^{2}$. The number of particle-hole excitations in each grain is of the order of $\nu\left(\epsilon_{F}\right) T \gg 1$, and the energy of a particle-hole is of the order of $T$. This gives us a rough estimate $\kappa(T) \propto g^{2} T^{3} / E_{c}^{2}$, in agreement with our detailed calculation. The near-continuum of states in the metallic grains means the electron-hole excitations can have very low energy; in this sense cotunneling in regular granular metals differs fundamentally from exciton transport in the Hubbard model even when intergrain tunneling is small. However there seems to be some resonance with excess heat transport by delocalized tightly-bound excitons in dirty semiconductors [21, 22]. Experimentally, $A l-G e$ granular systems in the $g \gg 1$ regime [26] show a linear $-T$ thermal conductivity with the value of the Lorenz number somewhat larger than $L_{0}$, $L \approx 1.8 L_{0}$ at the lowest temperatures measured. This is consistent with our assessment.
Our predictions can be experimentally verified: even though the $g^{2} T^{3} / E_{c}^{2}$ law we obtain for $\kappa(T)$ is reminiscent of the $T^{3}$ phonon contribution, it can be distinguished experimentally through its dependence on $g$ and $E_{c}$. Besides, the electronic contribution may be of the order of the phonon contribution as can be seen in the following rough estimate. Let $a$ denote the distance between grain centers, $l$ the phonon mean free path, $c$ the speed of sound, $n$ the atomic number density, and $\Theta_{D}$ the Debye temperature of the material. The ratio of the electronic and phononic thermal conductivities is then of the order of $r=\left(g \Theta_{D} / E_{c}\right)^{2}\left(k_{B} \Theta_{D}\right) /($ hancl $)$. In a typical three-dimensional granular metal, the grain diameter is of the order of $100 \AA$ and the insulating space between the grains is about $10 \AA$. With $\Theta_{D} \sim 300 \mathrm{~K}$, $E_{c} \sim 100 K, g \lesssim 1, a \sim 100 \AA, l \sim 10 \AA[27], c \sim 10^{3} \mathrm{~m} / \mathrm{s}$, and $n \sim 10^{28} \mathrm{~m}^{-3}$, we estimate $r \approx g^{2} \sim O(1)$. The electronic contribution can be further enhanced by increasing the size of the grains and choosing an insulator with a higher dielectric constant; each of these decreases $E_{c}$. Physically, the surprisingly large electronic thermal conductivity can be attributed to the large electron speed compared to the speed of sound.

We are grateful to D. E. Khmelnitskii for valuable discussions. V.T. thanks Trinity College, Cambridge for a JRF, and Y.L.L. thanks Trinity College and Cavendish Laboratory, Cambridge for support.

[1] G. H. Wiedemann and R. Franz, Ann. Phys., Leipzig,(2) 89, 497 (1853).

[2] G. V. Chester and A. Thellung, Proc. Phys. Soc. 77, 1005 (1961).

[3] M. J. Kearney and P. N. Butcher, J. Phys. C: Solid State Phys. 21, L265 (1988).

[4] L. Smrcka and P. Streda, J. Phys. C: Solid State Phys. 10, 2153 (1977).

[5] J. S. Langer, Phys. Rev. 128, 110 (1962).

[6] C. Castellani, C. DiCastro, G. Kotliar, P. A. Lee, and G. Strinati, Phys. Rev. B 37, 9046 (1988).

[7] D. V. Livanov, M. Y. Reizer, and A. V. Sergeev, JETP 72, 760 (1991).

[8] B. Arfi, J. Low Temp. Phys. 86, 213 (1992).

[9] D. R. Niven and R. A. Smith, Phys. Rev. B 71, 035106 (2005).

[10] G. Catelani and I. L. Aleiner, JETP 100, 331 (2005).

[11] R. Raimondi, G. Savona, P. Schwab, and T. Lück, Phys. Rev. B 70, 155109 (2004).

[12] I. S. Beloborodov, A. V. Lopatin, F. W. J. Hekking, R. Fazio, and V. M. Vinokur, Europhys. Lett. 69, 435 (2005).

[13] I. S. Beloborodov, K. B. Efetov, A. Altland, and F. W. J. Hekking, Phys. Rev. B 63, 115109 (2001).

[14] V. Ambegaokar, U. Eckern, and G. Schön, Phys. Rev. Lett. 48, 1745 (1982).

[15] A. Gerber, A. Milner, G. Deutscher, M. Karpovsky, and A. Gladkikh, Phys. Rev. Lett. 78, 4277 (1997).

[16] R. W. Simon, B. J. Dalrymple, D. Van Vechten, W. W. 
Fuller, and S. A. Wolf, Phys. Rev. B 36, 1962 (1987).

[17] Y. Ando, G. S. Boebinger, A. Passner, T. Kimura, and K. Kishio, Phys. Rev. Lett. 75, 4662 (1995).

[18] G. S. Boebinger, Y. Ando, A. Passner, T. Kimura, M. Okuya, J. Shimoyama, K. Kishio, K. Tamasaku, N. Ichikawa, and S. Uchida, Phys. Rev. Lett. 77, 5417 (1996).

[19] K. M. Lang, V. Madhavan, J. E. Hoffman, E. W. Hudson, E. Eisaki, S. Uchida, and J. C. Davis, Nature 415, 412 (2002).

[20] J. M. Luttinger, Phys. Rev. 135, A1505 (1964).

[21] R. Berkovits, Phys. Rev. B 60, 26 (1999).

[22] R. Berkovits and B. I. Shklovskii, J. Phys.: Condens.
Mat. 11, 779 (1999).

[23] G. Zárand, G. T. Zimányi, and F. Wilhelm, Phys. Rev. B 62, 8137 (2000).

[24] K. B. Efetov and A. Tschersich, Europhysics Lett. 59, 114 (2002).

[25] Y. L. Loh, V. Tripathi, and M. Turlakov, Phys. Rev. B 72, 233404 (2005).

[26] Y. Shapira and G. Deutscher, Phys. Rev. B 30, 166 (1984).

[27] The phonons are assumed to scatter randomly at the boundaries of the metallic and insulating regions, so their mean free path in the insulator, $l$, is also of the order of $10 \AA$. 